\title{
Enhanced Thermoelectric Properties of Armchair Graphene Nanoribbons with Pore Passivation
}

\author{
Sukhdeep Kaur ${ }^{1}$, Deep Kamal Kaur Randhawa ${ }^{2}$ and SukhleenBindra Narang ${ }^{3}$ \\ ${ }^{1 \& 3}$ Department of Electronics Technology, Guru Nanak Dev University, Amritsar, Punjab, India \\ ${ }^{2}$ Department of Electronics and Communication Engineering, Guru Nanak Dev University, RC Jalandhar, Punjab, India \\ E-Mail: gndusukhdeep@gmail.com,randhawadk@gmail.com, sukhleen2@yahoo.com
}

\begin{abstract}
There is a need to discover efficient thermoelectric materials that can generate electricity from waste heat and could play an important role in a global sustainable energy solution. Graphene Nanoribbons have been explored for a range of pore dimensions in order to achieve better thermoelectric performance. In this paper, we investigate the thermoelectric properties of porous armchair graphene nanoribbons by introducing hydrogen atoms as passivators at the pore surfaces. The aim of this work is to study the influence of pore passivation on the thermoelectric parameters as a function of pore geometry so as to open the possibility for an optimal pore engineering which can significantly improve the thermoelectric efficiency. The results show that the phonon thermal conductivity has a very little dependence on the pore edge passivation. An improvement in thermoelectric figure of merit is achieved due to the increased values of the power factor with consistent values of thermalconductivity. The unique thermoelectric properties of graphene nanoribbons with pore passivation suggest their great potentials for nanoscale thermoelectric applications. Within ballistic transport regime, semi-empirical extended Huckel method has been used for electrical properties while Tersoff potential has been employed for phononic calculations.
\end{abstract}

Keywords: Nanoribbon, Pore, Passivation, Thermoelectric

\section{INTRODUCTION}

In recent years, there has been growing interest in the search of thermo-electric materials due to their ability to convert dissipated heat into electricity which can be exploited to meet the energy demands of the society. ${ }^{1}$ The performance of thermo-electric material depends on its electronic and phononic properties. It is given in terms of the dimensionless figure of merit, $Z T=\frac{S^{2} G T}{k e+k p h}$ where $S$ is Seebeck Coefficient, Gis electrical conductance and $T$ is temperature. The thermal conductance is composed of two parts, thermal electron conductance $k_{e}$, and thermal phonon conductance $\mathrm{k}_{\mathrm{ph}}$. For efficient energy conversion, ZT needs to have a high value which in turn means higher values of $S$ and $\mathrm{G}$ while lower values of $\mathrm{k}$.

Graphene, a mono-layer of graphite with a honey-comb lattice exhibits distinguished physical properties such as remarkably high thermal conductivity and high mobility. ${ }^{2-}$ ${ }^{3}$ However, being a zero band gap material, it has a very small value of Seebeck Co-efficient which limits its use in thermoelectric applications. An enhancement of thermoelectric properties can be achieved by nanostructuring graphene ${ }^{4}$ into graphene nanoribbons (GNRs) with reduced thermal conductivity. For further reducing the thermal conductivity, several approaches such as including defects ${ }^{5}$ andedge passivation ${ }^{2}$ because of their increased phonon scattering have been considered.

Recently, nanopore engineering ${ }^{6}$ has come into role which reduces the $\mathrm{k}_{\mathrm{ph}}$ and hence increases ZT. Several studies have been carried out to investigate the performance of porous graphene nanoribbons. ${ }^{6-8}$ Our previous work suggested an increase in the thermoelectric performance with the increase in the pore size. ${ }^{8}$

G. A. Nemnesa et al. ${ }^{3}$ studied the thermal properties of graphene nanoribbons passivated with halogen atoms and concluded that there occurs a systematic decrease of the scaled thermal conductanceas the halogen atomic number is increased. This effect is further amplified with the enlargement of the pore size. Lin Huet et al. ${ }^{9}$ theoretically investigated the thermal transport properties of graphene nanomeshes with hydrogen passivated circular pores and concluded that the thermal conductivity has a very weak dependence on the pore edge passivation. J. Hu et $a .^{2}$ predicted that the edge $\mathrm{H}$ passivation considerably reduces the thermal conductivity of graphene nanoribbons.

Thus, there has been an extensive research on the thermal transport of pore passivated structures. But the systematic study of the overall thermoelectric figure of merit which includes the electron transport as well to evaluate the overall figure of merit has beenlacking. Therefore, the purpose of this article is to investigate the overall thermoelectric properties of pore passivated GNRs characterized by different pore sizes, pore morphology and varying temperature. In this work, non-equilibrium Green's function method combined with the Extended Huckel Theory has been employed while Hydrogen is used as the passivator because of its ability to stabilize the lonely atoms and hence to enhance the thermodynamic stability of the nanoribbons.

\section{MODELLING AND COMPUTATIONAL METHODS}

The atomistic model of our work is armchair graphene nanoribbon with pore edge passivated with hydrogen atoms as shown in Fig 1. It consists of three regions:left and right 
semi-infinite thermal leads and a centralregion.Here, we mainly focus on the ballistic transport, while theinelastic effects such as electron-phonon and phonon-phonon interactions are neglected.

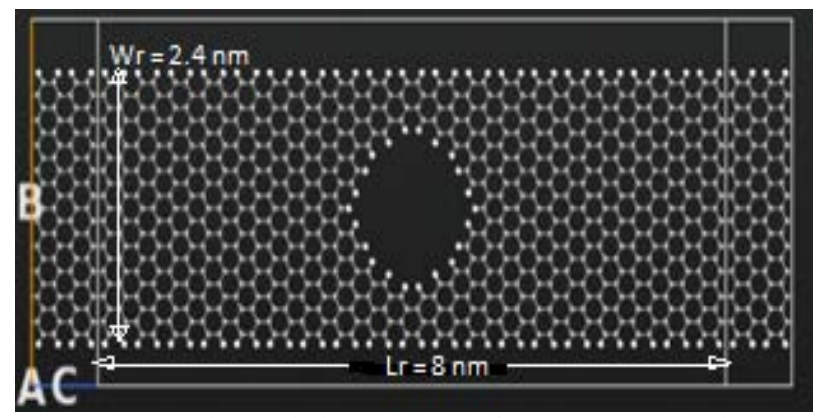

Fig. 1 Geometrical optimized structure of the graphene nanoribbon incorporating a circular pore of $1.48 \mathrm{~nm}$ diameter with pore edges passivated with hydrogen atoms

According to Extended Huckel theory, the retarded Green function $G^{R}=\left(E S_{o v}-H-\Sigma_{L}-\Sigma_{R}\right)^{-1}$ where $E$ is the electron energy. Here, the Hamiltonian Hand overlap matrix $\boldsymbol{S}_{\boldsymbol{o v}}$ of the central region are calculated from extended Huckel technique $^{10-11}$ using Hoffman basis. Using non-equilibrium Green function (NEGF),the electron transmission function $\mathrm{T}(\mathrm{E})$ can be obtained through:

$$
T(E)=\text { Trace }\left[\Gamma_{R}(E) G^{R}(E) \Gamma_{L}(E) G^{R \dagger}(E)\right] \text {. }
$$

In this expression, $\Gamma_{L, R}(E)=i\left(\Sigma_{L, R}(E)-\Sigma_{L, R}(E)\right)$ denotes the level broadening due to the coupling between the central scattering region and the electrodes while $\Sigma_{L, R}(E)$ are the retarded self-energies for the electrodes. The mesh cut-off and mesh points were set at 10 Hartree and $1 \times 1 \times 100 \mathrm{k}$ points at a temperature of $300 \mathrm{~K}$.

The transport properties Seebeck Coefficient $S$, electrical conductance $G$ and electronic thermal conductance $k_{e}$ can be obtained in the linear response regime asfollows ${ }^{6}$ :

$$
\begin{aligned}
& G=e^{2} L_{0 .} \\
& S=\frac{L_{1}}{e T L_{0}} . \\
& k_{e}=\frac{L_{2}-L_{1}^{2} / L_{0}}{T} .
\end{aligned}
$$

where the $\mathrm{n}^{\text {th }}$ order Lorenz function is given by:

$$
L_{n}=\frac{2}{h} \int d E T(E)\left(-\frac{\partial f}{\partial E}\right)(E-\mu)^{n} .
$$

In the above equation, $e$ is the elementary charge, $f=1+$ exp $\left.\left((E-\mu) / k_{B} T\right)\right)^{-1}$ is the Fermifunction with $\partial f / \partial E$ being its derivative ${ }^{12}$ where $k_{B}$ is the Planck constant, $\mu$ is the chemical potential and $T$ is the average temperature.

Here, theLandauer approach ${ }^{13}$ has been used to calculate the phonon thermal conductance as follows:

$$
k_{p h}=\frac{h^{2}}{2 \pi k_{B} T^{2}} \int d \omega \omega^{2} T_{p h}(\omega)\left(\frac{\partial n}{\partial \omega}\right) \text {. }
$$

For phonon transmission $T_{p h}(\omega)$ is calculated in the same manner as that of electrical conductance. Hhas been substituted by a force constant matrix Kwhich has been calculated usingempirical Tersoff potential (Tersoff_CH_2005) while S has been substituted with a diagonal matrix of atomic mass $\mathrm{M}$ respectively. Here, the factor $\partial n / \partial \omega$ is the derivative of the Bose-Einstein distribution for phonons.

Virtual NanoLab-Atomistix Toolkit (ATK) software package has been used to carry out the phonon and electron simulations.In order to minimise the forces on individual atoms to be smaller than $0.01 \mathrm{eV}^{-1}$, each structure has been optimized before transport calculations with its atom coordinates relaxed.Here, only independent electron and phonon calculations are carried outwhile neglecting the electron-phonon interactions on the basis of work done by Chenet al. ${ }^{14}$ where they have experimentally showed that the mean free path for electron-acoustic phonon scattering in graphene is greater than $2 \mu \mathrm{m}$, which is much larger than our proposed model dimensions.

\section{RESULTS AND DISCUSSION}

\section{A. Pore Size Dependence}

The width $\mathrm{W}_{\mathrm{r}}$ andlength $\mathrm{L}_{\mathrm{r}}$ of the ribbon as shown in Fig1 is kept constant at $2.4 \mathrm{~nm}$ (21 atoms wide) by $8 \mathrm{~nm}$ while the pore dimension is varied at $0.98 \mathrm{~nm}, 1.23 \mathrm{~nm}, 1.48 \mathrm{~nm}$ and $1.72 \mathrm{~nm}$.

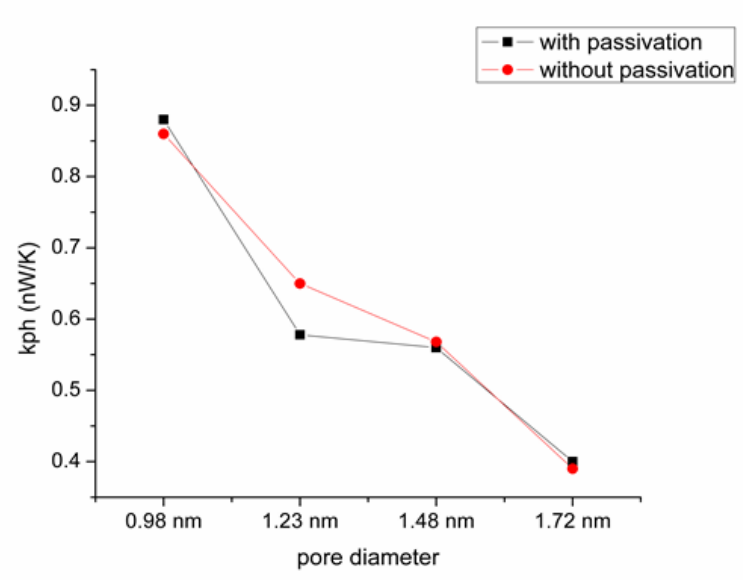

Fig. 2 Phonon Thermal Conductivity dependence of pore passivated structure plotted against the diameter of the pore. The respective values for non-passivated structure have been shown for reference

As illustrated in the Fig 2, phonon thermal conductivity is almost independent of pore passivation for the different pore sizes except for a diameter of $1.23 \mathrm{~nm}$ (corresponding to the removal of 72 atoms from the centre of the nanoribbons) where it is found to decrease by $12 \%$ as compared with the structure with unpassivated pore edges. Here the same geometrical structure but with unpassivated pores has been taken as reference. Fig 3(a-d) shows the chemical potential dependence of $\mathrm{S}, \mathrm{G}, \mathrm{k}_{\mathrm{e}}$ and ZT for the structures respectively. 

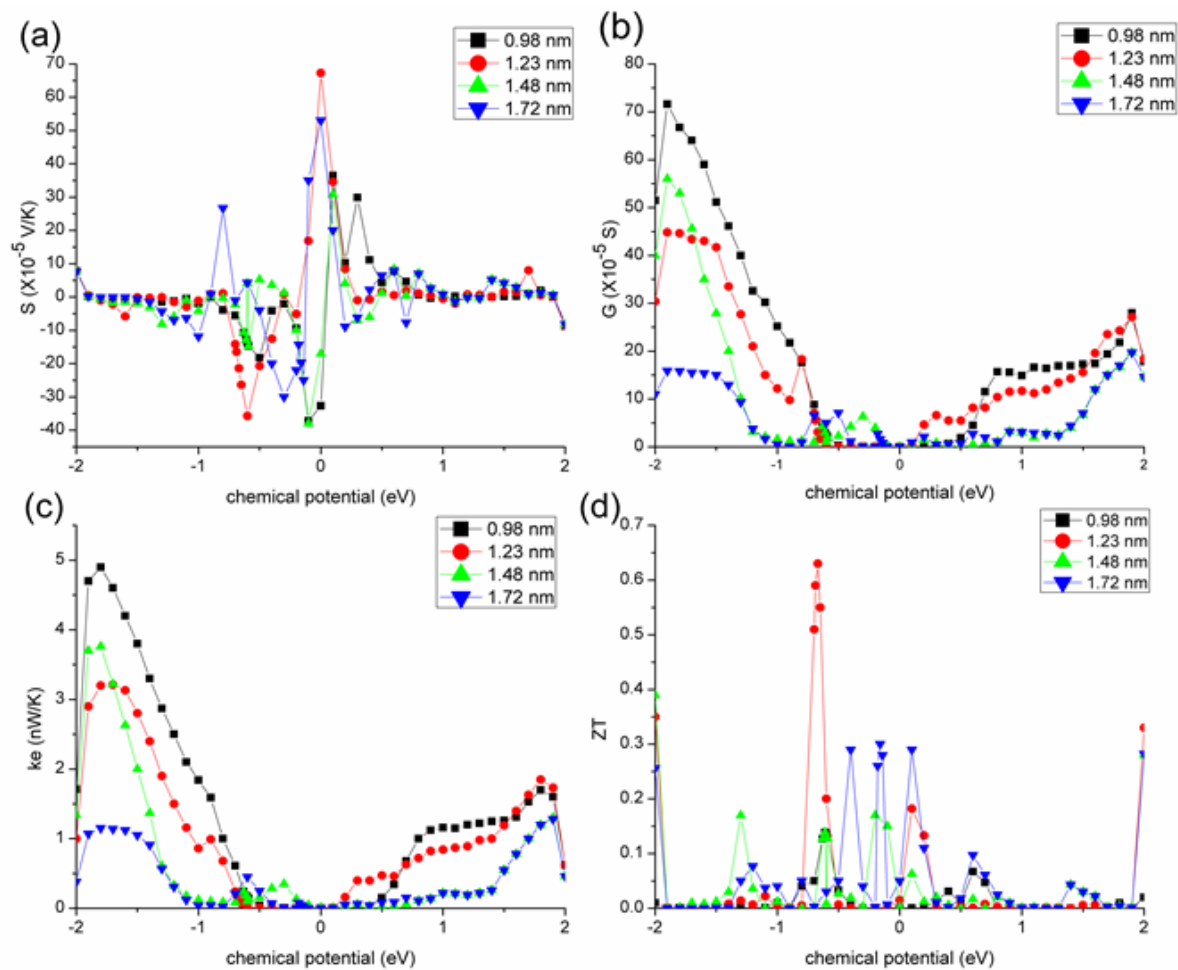

Fig. 3 Chemical potential dependence of (a)Seebeck Coefficient, (b) Conductance, (c) Electron Thermal Conductance and (d) Figure of merit ZT respectively

To compute the effect of electron transport, we find the position of the chemical potential at which ZT is maximum and then extract the values of $S, G$ and $k_{e}$. Since the $k_{e}<<$ $\mathrm{k}_{\mathrm{ph}}$, therefore the phonon thermal conductance is the dominant factor in deciding the overall thermal conductance. With the introduction of passivators, the $\mathrm{S}$ and $\mathrm{G}$ are improved which in turn improves the power factor for all pore sizes as shown in Fig4(a-d).
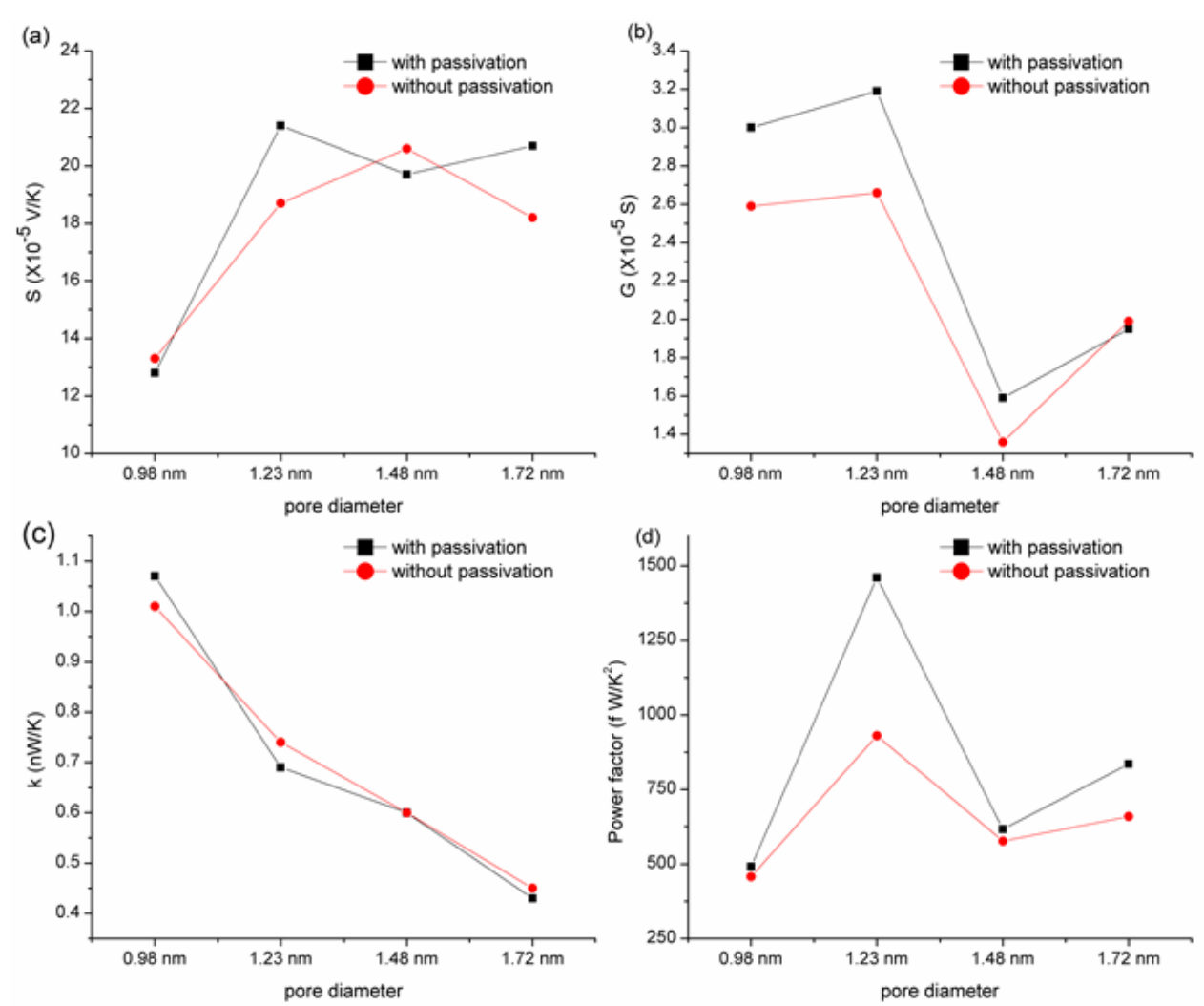

Fig. 4 (a) Seebeck Coefficient, (b) Conductance, (c) Thermal Conductance and (d) Power Factor plotted against the diameter of the pore 
As a result of this, $\mathrm{ZT}$ is found to improve with the maximum increase in the case of $1.23 \mathrm{~nm}$ as illustrated in Fig 5.

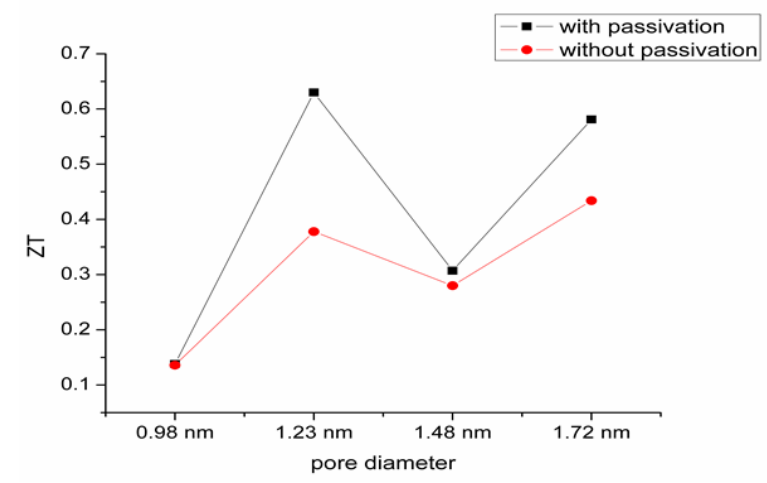

Fig. 5 Figure of merit of pore passivated structure plotted against the diameter of pore. The non-passivated values are shown for comparison

\section{B. Pore Shape Dependence}

In addition, the effect of change in the pore shape is also investigated. The number of atoms deleted from the ribbon for the fabrication of $0.98 \mathrm{~nm}$ and $1.48 \mathrm{~nm}$ diameter porous nanoribbons are 34 and 72 respectively. Considering the equivalent number of atoms, pore shapes of rhombus and triangle are also considered. The choice for these particular numbers is done on the basis of the feasibility of fabrication to result in a symmetrical pore geometry which means an

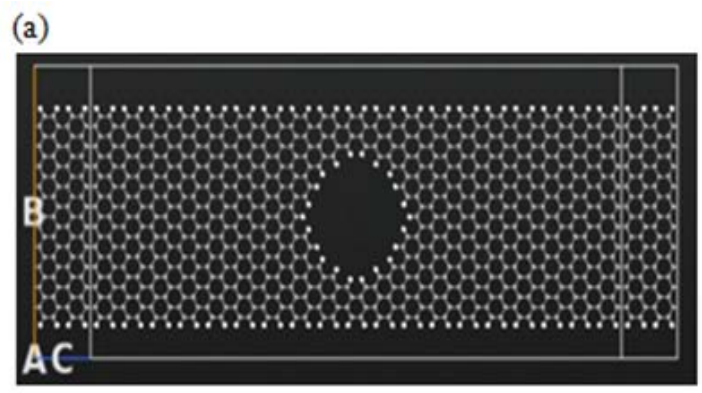

(c)

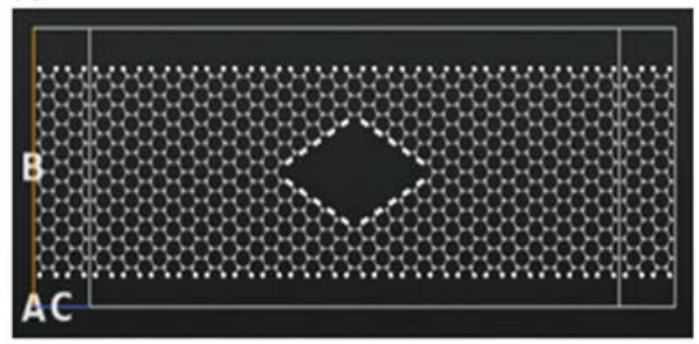

Fig. 7 Optimised view of (a) circular (b) triangular and (c) rhombus shape corresponding to the removal of 72 atoms

But on the other hand, it is observed that the Seebeck coefficient is most favourable in case of circular shape because of its ability to efficiently modulate the transmission spectrum for optimum thermoelectric performance. $^{8}$ Therefore, circular shape is the most preferred choice for pore passivated AGNRs for an efficient thermoelectric performance. equal number of side atoms both above and below the pore at the centre.

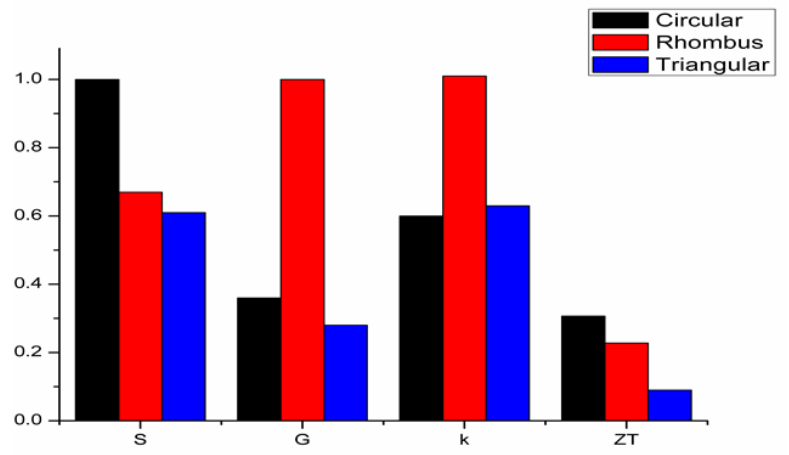

Fig. 6 Comparison of transport co-efficients for circular, triangular and rhombus shaped pore passivated graphene nanoribbon corresponding to removal of 72 atoms. The peak values have been normalized to one while the other values are normalized relatively

Fig. 6 shows the comparison in transport coefficients for the different shapes. The peak values have been normalised to one while the other values are normalised relatively. Amongst these three shapes, the electron conductance and the thermal conductivity is found to be maximum in case of rhombus shape. This can be attributed to the more number of side channels $\mathrm{N}_{\mathrm{c}}$ (above and below the pore) as shown in Fig7 where the removal of 72 atoms results in $\mathrm{N}_{\mathrm{c}}=4$ in case of circular and triangular shape while an $\mathrm{N}_{\mathrm{c}}=5$ in case of rhombus shape.

\section{(b)}

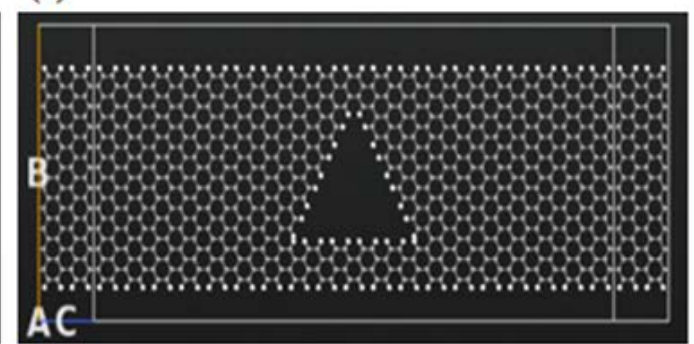



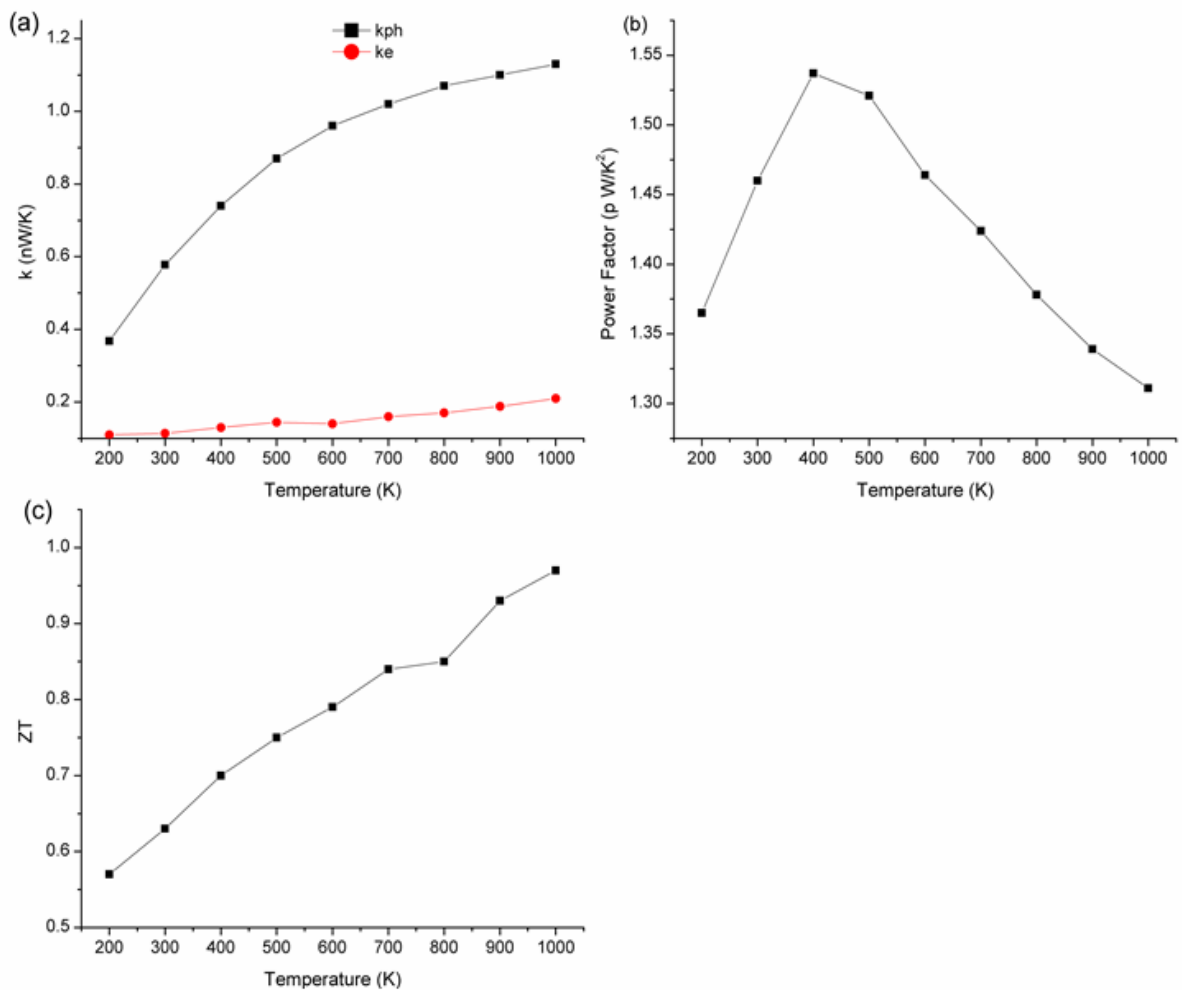

Fig. 8 Temperature dependent behaviour of (a) Thermal conductance (b) Power Factor and (c) Figure of Merit ZT

This is because of the significant increase in population of the phonon modes above the minima of the phononic transmission function. ${ }^{3}$ On the other hand, power factor remains almost constant as shown in Fig. 8(b). However, it is the presence of temperature term in the numerator of $\mathrm{ZT}$ which contributes to an improvement as shown in Fig 8(c) inspite of an increase in $\mathrm{k}_{\mathrm{ph}}$. The electron-phonon and electron-electron interactions which dominate at high temperatures are not being taken into account while performing the calculations.

\section{CONCLUSION}

In summary, we have investigated the thermoelectric properties of porous graphene nanoribbons with hydrogen passivated pore surfacessubjected to different pore geometries. It is found that the pore passivation resulted in an improvement of thermoelectric figure of merit for the different pore sizes. The physical mechanism responsible for this effect is the favourable electron transport supported with almost same or reduced values of thermal conductivity for a particular pore size. The hydrogen passivation effect further improves with increasing temperature. Out of the different pore shapes, the circular shape is found to be the most preferred choice for appealing thermoelectric applications. These results indicate the possibility of tuning both the electronic and thermal properties by hydrogen passivation with an aim to achieve efficient thermoelectric devices.

\section{REFERENCES}

[1] D. L. Nika and A. Balandin, J.Phys.:Condens. Mat, Vol. 24, pp. 203233, 2012.

[2] J. Hu, S. Schiffli, A. Vallabhaneni, X. Ruan and Y. P. Chen, Appl. Phys. Lett., Vol. 97, pp. 107-133, 2010.

[3] G. A. Nemnes, C. Visan and A. Manolescu, J. Mater. Chem. C., Vol.5, pp. 35-44, 2017.

[4] D. Dragoman and M.Dragoman, Appl. Phys. Lett., Vol. 91, 116-203, 2007.

[5] J. Haskins, A. Kinaci, C. Sevik, H. Sevincli, G. Cuniberti, and T. Cagin, ACS Nano, Vol. 5, pp. 73-79, 2011.

[6] M. S. Hossain, F. Al-Dirini, F.M.Hossain and E.Skafidas, Sci. Rep, Vol. 5, pp. 97-112, 2015.

[7] H. Sadeghi, S. Sangtarash and C.J. Lambert, Beilstein J. Nanotech., Vol. 6, pp. 1176, 2015.

[8] S. Kaur, S. B. Narang and D. K. Randhawa, J .Mater. Res., Vol.32, pp. 1149, 2017.

[9] L. Hu and D.Maroudas, J. Appl.Phys., Vol.116, pp. 184-194, 2014.

[10] D. Kienle, J.I.Cerda and A.W.Ghosh, J. Appl. Phys., Vol. 100, pp. 43-47, 2006.

[11] K.Stokbro, D. E. Peterson, S. Smidstrup, A. Blom, M. Ipsen and K.Kaasbjerg, Phys. Rev. B., Vol. 82, pp. 75-78, 2010.

[12] K. Esfarjani, M. Zebarjadi and Y. Kawazoe,Phys. Rev. B: Condens. Matter Mater. Phys., Vol.73, pp. 403-406, 2006.

[13] R. Landauer, IBM. J. Res. Dev., Vol. 1, pp. 223, 1957.

[14] J. H. Chen, C. Jang, S.Xiao, M. Ishigami and M.S. Fuhrer, Nat. Nano, Vol. 3, pp. 206, 2008. 